\title{
The puzzling behavior of HNCO isomers in molecular clouds ${ }^{\star}$
}

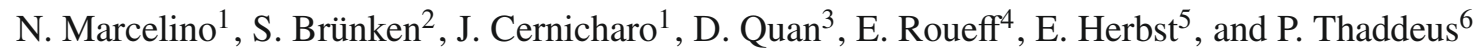 \\ ${ }^{1}$ Centro de Astrobiología (CSIC-INTA), Laboratorio de Astrofísica Molecular, Ctra de Ajalvir Km 4, 28850, Torrejón de Ardoz, \\ Madrid, Spain \\ e-mail: nmarcelino@cab.inta-csic.es; jcernicharo@cab.inta-csic.es \\ 2 I. Physikalisches Institut, Universität zu Köln, Zülpicher Str. 77, 50937 Köln, Germany \\ e-mail: bruenken@ph1.uni-koeln.de \\ 3 Chemical Physics Program, The Ohio State University, Columbus, OH 43210, USA \\ e-mail: dquan@chemistry.ohio-state.edu \\ 4 Observatoire de Paris-Meudon, LUTH UMR 8102, 5 Place Jules Janssen, F-92195 Meudon Cedex, France \\ e-mail: evelyne.roueff@obspm.fr \\ 5 Departments of Physics, Astronomy, and Chemistry, The Ohio State University, Columbus, OH 43210, USA \\ e-mail: herbst@mps .ohio-state.edu \\ ${ }^{6}$ Harvard-Smithsonian Center for Astrophysics, 60 Garden St., Cambridge, MA 02138, USA \\ e-mail: pthaddeus@cfa.harvard.edu
}

Received 4 December 2009 / Accepted 26 March 2010

\section{ABSTRACT}

\begin{abstract}
Context. Isocyanic acid (HNCO) has been observed in different physical environments in the interstellar medium (ISM) and in external galaxies. $\mathrm{HNCO}$ has several metastable isomers with a ground electronic singlet state: HOCN, HCNO, and HONC. The recent detection of fulminic acid (HCNO) in prestellar and protostellar cores and cyanic acid (HOCN) in warm molecular sources (e.g. hot cores) in the Galactic center proves that these species could also be common constituents of the ISM.

Aims. To shed some light on the possible formation pathways of these species, we searched for HCNO in the sources where HOCN has been previously detected and vice versa. We have also included the low-mass protostar IRAS 16293-2422, where HNCO is found to be prominent.

Methods. Using the new EMIR receivers at the IRAM 30-m telescope, we performed deep searches for three rotational transitions of $\mathrm{HOCN}$ and four of $\mathrm{HCNO}$.

Results. We report the detection of HOCN in four sources - three dense cores and the lukewarm corino L1527 - where HCNO has been previously observed. HOCN is tentatively detected toward the protostellar binary IRAS 16293-2422. However, HCNO has been detected neither in this source nor in the sources of the Galactic center where HOCN has been previously reported. The derived abundance ratios $\mathrm{HCNO} / \mathrm{HOCN}$ are close to unity in quiescent clouds, while they are less than $0.01-0.1 \mathrm{in}$ warm clouds. We attempt to explain these results by using both gas-phase and gas-grain chemical models.
\end{abstract}

Key words. astrochemistry - line: identification - ISM: abundances - ISM: clouds - ISM: molecules

\section{Introduction}

Isomeric forms of molecular species are of interest in astrochemistry because they can help constrain the chemical processes leading to their formation and depletion, whether they occur in the gas phase or on the surfaces of dust particles. Furthermore, for some isomers, the stablest one is not necessarily the most abundant in molecular clouds, as is the case for HCN and HNC in cold sources. This pair of isomers, for which gas phase chemical models predict similar abundances at low temperature (see Herbst et al. 2000, and references therein), show however a peculiar behavior depending on the kinetic temperature of the source. Whereas the abundance of the metastable isomer HNC is similar to, and even higher than, that of $\mathrm{HCN}$ in dark cloud cores, in warm molecular clouds the abundance ratio $\mathrm{HNC} / \mathrm{HCN}$ is much less than unity (Irvine \& Schloerb 1984; Churchwell et al. 1984; Schilke et al. 1992; Hirota et al. 1998). Indeed, the HCN abundance does not change considerably between cold and warm

* This work was based on observations carried out with the IRAM $30-\mathrm{m}$ telescope. IRAM is supported by INSU/CNRS (France), MPG (Germany) and IGN (Spain). clouds, but the HNC abundance decreases. This is puzzling since both isomers are mainly formed from the dissociative recombination of $\mathrm{HCNH}^{+}$. According to Watson \& Walmsley (1982), these observational results require either a production mechanism that produces more $\mathrm{HNC}$ than $\mathrm{HCN}$ and a destruction process that primarily destroys $\mathrm{HNC}$ when the temperature increases, or a more effective isomerization process in clouds with higher temperatures. Regarding the last point, Talbi et al. (1996) showed that the $\mathrm{HNC} / \mathrm{HCN}$ isomerization reactions with atomic hydrogen indeed become more effective at higher temperatures owing to activation energy barriers in both directions. Thus, the relative abundance of different isomers in molecular clouds is probably not thermodynamic but kinetic in origin.

HNCO has several metastable isomers with a singlet ground electronic state: HOCN, HCNO, and HONC. These isomers lie at increasingly higher energy than $\mathrm{HNCO}$ according to quantum calculations $\left(24.7,70.7\right.$, and $84.1 \mathrm{kcal} \mathrm{mol}^{-1}$, respectively; see Schuurman et al. 2004). Since there is not an obvious common precursor for them, their relative abundances in molecular clouds might provide some clues concerning the respective contributions of gas-phase and grain processes to the chemistry. 
Table 1. Observed sources.

\begin{tabular}{lccc}
\hline \hline Source & $\begin{array}{c}\text { RA } \\
(\mathrm{J} 2000.0)\end{array}$ & $\begin{array}{c}\text { Dec } \\
(\mathrm{J} 2000.0)\end{array}$ & $\begin{array}{c}v_{\text {LSR }} \\
\left(\mathrm{km} \mathrm{s}^{-1}\right)\end{array}$ \\
\hline B1-b & $03^{\mathrm{h}} 33^{\mathrm{m}} 20.8^{\mathrm{s}}$ & $+31^{\circ} 07^{\prime} 34.0^{\prime \prime}$ & 6.7 \\
L1527 & $04^{\mathrm{h}} 39^{\mathrm{m}} 53.9^{\mathrm{s}}$ & $+26^{\circ} 03^{\prime} 11.0^{\prime \prime}$ & 5.9 \\
TMC-1(CP) & $04^{\mathrm{h}} 41^{\mathrm{m}} 41.9^{\mathrm{s}}$ & $+25^{\circ} 41^{\prime} 27.1^{\prime \prime}$ & 6.0 \\
L1544 & $05^{\mathrm{h}} 04^{\mathrm{m}} 18.1^{\mathrm{s}}$ & $+25^{\circ} 10^{\prime} 48.0^{\prime \prime}$ & 7.2 \\
L183 & $15^{\mathrm{h}} 54^{\mathrm{m}} 08.6^{\mathrm{s}}$ & $-02^{\circ} 52^{\prime} 10.0^{\prime \prime}$ & 2.4 \\
IRAS 16293-2422 & $16^{\mathrm{h}} 32^{\mathrm{m}} 22.6^{\mathrm{s}}$ & $-24^{\circ} 28^{\prime} 33.0^{\prime \prime}$ & 4.0 \\
SgrB2N & $17^{\mathrm{h}} 47^{\mathrm{m}} 20.0^{\mathrm{s}}$ & $-28^{\circ} 22^{\prime} 19.0^{\prime \prime}$ & 64 \\
SgrB2M & $17^{\mathrm{h}} 47^{\mathrm{m}} 20.4^{\mathrm{s}}$ & $-28^{\circ} 23^{\prime} 07.0^{\prime \prime}$ & 62 \\
SgrB2S & $17^{\mathrm{h}} 47^{\mathrm{m}} 20.5^{\mathrm{s}}$ & $-28^{\circ} 23^{\prime} 45.0^{\prime \prime}$ & 61 \\
SgrB2M-B & $17^{\mathrm{h}} 47^{\mathrm{m}} 21.9^{\mathrm{s}}$ & $-28^{\circ} 21^{\prime} 27.0^{\prime \prime}$ & 68 \\
\hline
\end{tabular}

Two isomeric forms of HNCO have recently been detected in space, HCNO (Marcelino et al. 2009) and HOCN (Brünken et al. 2010). Fulminic acid (HCNO) is observed in three dark cloud cores and toward the low-mass star-forming region L1527, with an abundance ratio of $\mathrm{HNCO} / \mathrm{HCNO}$ between 20 and 60 . However, HCNO has not been detected toward the direction of the cyanopolyyne peak of TMC-1 or in the Orion Hot Core region. Cyanic acid (HOCN) is detected in several positions in the giant molecular cloud SgrB2 (Brünken et al. 2009a, 2010), after its characterization in the laboratory in the $\mathrm{cm}$ - and $\mathrm{mm}$ wave bands and a tentative detection in $\operatorname{SgrB} 2(\mathrm{OH})$ based on previous spectral line surveys (Brünken et al. 2009a). The reported $\mathrm{HNCO} / \mathrm{HOCN}$ abundance ratio in the Galactic center (GC) clouds is between 40-400. Brünken et al. (2009a) also obtained a tentative detection of HOCN in TMC- 1 through the fundamental transition at $20.9 \mathrm{GHz}$. The derived column density is $7 \times 10^{10} \mathrm{~cm}^{-2}$ and the HNCO/HOCN abundance ratio is $\sim 100$.

The chemistry of the CHNO isomers, where the term refers to all four species, seems to be rather complex. While in Marcelino et al. (2009), the observed abundances of HCNO and HNCO were reproduced well with a steady-state, gas-phase model, Brünken et al. (2009a, 2010) proposed models with both gas-phase and grain-surface chemistry to explain the isomeric abundances of HOCN and HNCO in the molecular clouds of the GC. In this paper we report observations of HOCN and HCNO toward different sources in order to shed some light on the possible formation pathways of these species in both the gas phase and on dust surfaces. We looked for HCNO in several positions in SgrB2 associated with hot or warm environments, where HOCN had previously been detected (see Brünken et al. 2010), while HOCN was searched for in the cold to lukewarm dense cores observed in Marcelino et al. (2009). We have also included in our search the well-studied low-mass protostar IRAS 16293-2422, where HNCO has been found to be prominent (Bisschop et al. 2008), and a rich organic chemistry has been widely observed as a result of mantle grain desorption and, possibly, warm gas chemistry (see, e.g., Cazaux et al. 2003; Kuan et al. 2004; Garrod \& Herbst 2006; Garrod et al. 2008, and references therein). The observed positions are shown in Table 1.

\section{Observations}

The observations were performed with the IRAM 30-m telescope (Granada, Spain) in April and May 2009. We used the new EMIR receivers at 3, 2, and $1 \mathrm{~mm}$ in combinations E090/E150 and E090/E230 (3 and $2 \mathrm{~mm}, 3$ and $1 \mathrm{~mm}$, respectively). All receivers were tuned in single-side band mode with image rejections of $13 \mathrm{~dB}$, and with both polarizations at the same
$\mathrm{T}_{\mathrm{MB}}(\mathrm{K})$
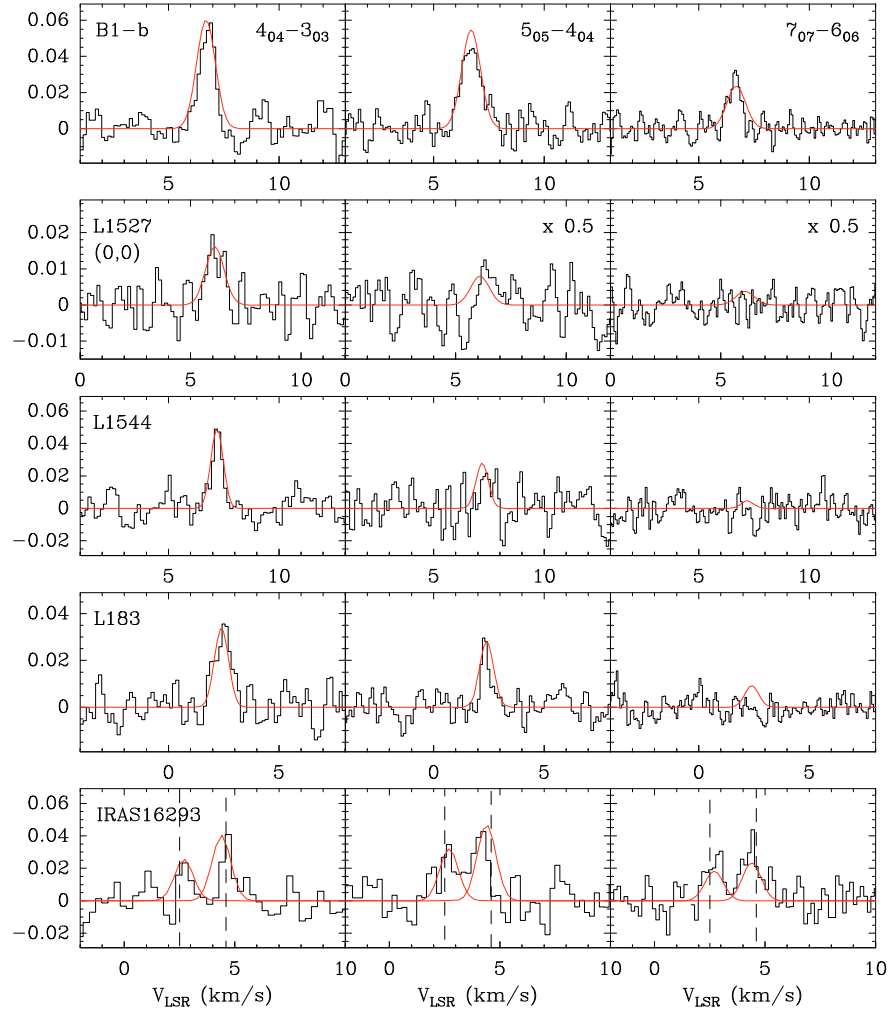

Fig. 1. Line profiles for the observed transitions of HOCN (black solid lines) and simulation from LVG calculations (red solid lines). Upper limits of the intensity scale for the central position of L1527 (second row of panels) have been reduced by the factor 0.5. For IRAS 162932422 (lowest panels), vertical dashed lines show the observed velocity components (see text).

frequency. System temperatures were between $70-130 \mathrm{~K}$ at 3 and $2 \mathrm{~mm}$ (with the highest value reflecting worse weather conditions) and $\sim 180 \mathrm{~K}$ at $1 \mathrm{~mm}$, except for the HCNO $\left(5_{05}-4_{04}\right)$ transition at $114 \mathrm{GHz}$, where the temperature was higher owing to the poorer atmospheric transmission $\left(T_{\text {sys }} \sim 300 \mathrm{~K}\right)$. Intensity calibration was performed using two absorbers at different temperatures. The atmospheric opacity at $225 \mathrm{GHz}$, obtained from the measurement of the sky emissivity and the use of the ATM code (Cernicharo 1985), was typically $\lesssim 0.1$ at $1 \mathrm{~mm}$ (water vapor column $1-3 \mathrm{~mm}$ ) during night time, and between $0.1-0.2$ in the afternoon (water vapor column $\sim 5 \mathrm{~mm}$ ).

Observing modes and spectral resolutions were selected depending on the sources. For the cold cores, we observed in frequency switching mode, since the lines are sufficiently narrow, while we used the wobbler switching mode for IRAS 162932422 and SgrB2. The off positions were checked to be free of HCNO and HOCN emission. The autocorrelator VESPA was used as a backend, with $40 \mathrm{kHz}\left(\sim 0.08-0.14 \mathrm{~km} \mathrm{~s}^{-1}\right), 80 \mathrm{kHz}$ $\left(\sim 0.11-0.28 \mathrm{~km} \mathrm{~s}^{-1}\right)$, and $320 \mathrm{kHz}\left(\sim 0.45-1 \mathrm{~km} \mathrm{~s}^{-1}\right)$ of resolution for the dense cores, IRAS 16293-2422, and SgrB2, respectively.

Pointing and focus were checked regularly (every 1.5 and $3 \mathrm{~h}$, respectively) on strong and nearby sources. At the observed frequencies, the beamwidth of the antenna is in the range $29^{\prime \prime}-17^{\prime \prime}$ and the main beam efficiency is $0.78-0.64$. All spectra have been calibrated in main beam temperature scale. The observed line profiles for HOCN are shown in Fig. 1 and the 
Table 2. HOCN observed line parameters.

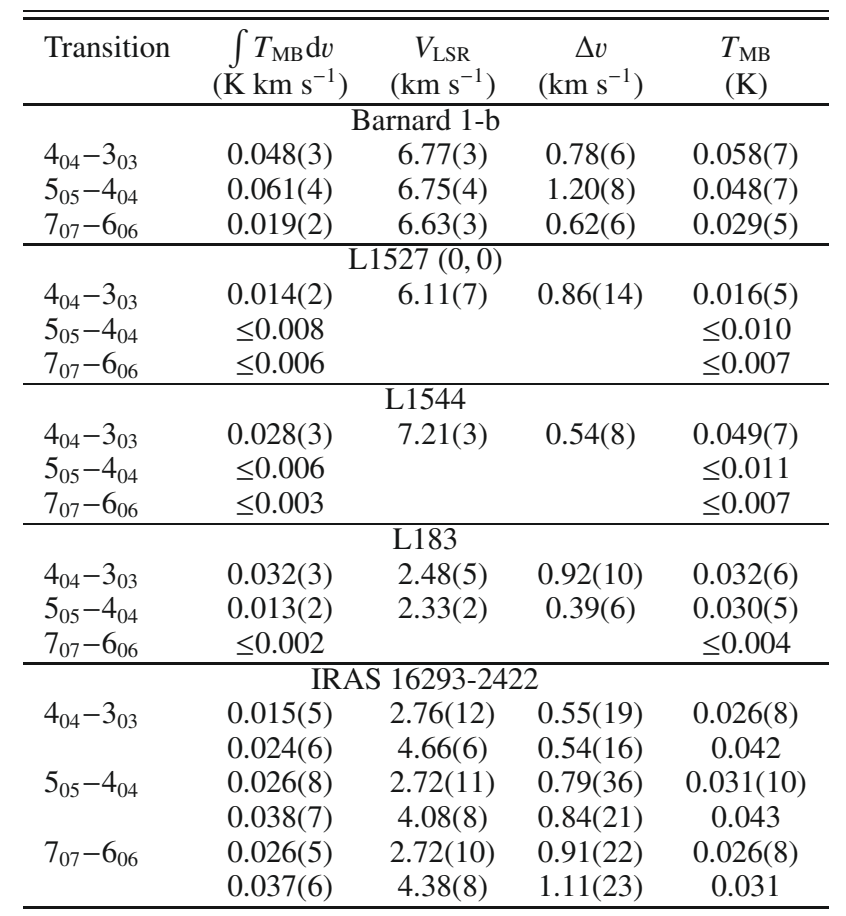

Notes. Numbers in parentheses are $1 \sigma$ uncertainties in units of the last digits. For non-detections, the upper limit to the integrated intensity $(1 \sigma)$ and the observed rms are shown. Adopted rest frequencies (MHz) for the $4_{04}-3_{03}, 5_{05}-4_{04}$, and $7_{07}-6_{06}$ transitions are 83900.572 , 104874.679 , and 146820.687, respectively, and their corresponding upper energy levels (K) are 10, 15, and 28 (Brünken et al. 2009a). For IRAS 16293-2422, line parameters were obtained for both velocity components (see text).

derived line parameters, obtained from Gaussian fits using the GILDAS package ${ }^{1}$, are shown in Table 2.

A total of $50 \mathrm{~h}$ were devoted to completion of the whole project. Three and four molecular transitions arising from $\mathrm{HOCN}$ and $\mathrm{HCNO}$, respectively, were observed in different sources. Owing to the expected low intensity (few tens of $\mathrm{mK}$ ) of the HOCN lines in dark clouds, we performed deep integrations on them. The lowest energy observed transition - the $4_{04}-3_{03}$ $\left(v=83900.572 \mathrm{MHz}, E_{\mathrm{u}}=10 \mathrm{~K}\right)$ line - was searched for in all dense cores shown in Table 1 (including a second position for L1527) for 2.5 to $5 \mathrm{~h}$ of on-source observing time. In those cores where this transition was detected - B1-b, L1527 $(0,0), \mathrm{L} 1544$, and L183 - we also searched for the $5_{05}-4_{04}$ $\left(v=104874.679 \mathrm{MHz}, E_{\mathrm{u}}=15 \mathrm{~K}\right)$ line for 2 to $4 \mathrm{~h}$ per position. We observed the $7_{07}-6_{06}\left(v=146820.687 \mathrm{MHz}, E_{\mathrm{u}}=28 \mathrm{~K}\right)$ line simultaneously with the former or the latter transition, resulting in a total observing time per source of 5-8 h. Toward IRAS 16293-2422, we used $2 \mathrm{~h}$ of observing time each for the HOCN $4_{04}-3_{03}$ and $5_{05}-4_{04}$ lines, and $4 \mathrm{~h}$ for the $7_{07}-6_{06}$ transition. Four HCNO lines - namely the $4-3(v=91751.320 \mathrm{MHz}$, $\left.E_{\mathrm{u}}=11 \mathrm{~K}\right), 5-4\left(v=114688.383 \mathrm{MHz}, E_{\mathrm{u}}=16 \mathrm{~K}\right), 6-5(v=$ $\left.137624.934 \mathrm{MHz}, E_{\mathrm{u}}=23 \mathrm{~K}\right)$, and $9-8(v=206430.497 \mathrm{MHz}$, $\left.E_{\mathrm{u}}=50 \mathrm{~K}\right)$ transitions - were searched for in several positions toward SgrB2 (see Table 1), using $\sim 1 \mathrm{~h}$ per position and transition. To compare our intensities with the previous HOCN observations (Brünken et al. 2010), we observed the $4_{04}-3_{03}$ and $7_{07}-6_{06}$ lines in all SgrB2 positions. For that purpose, we used

${ }^{1}$ http://wWw.iram. fr/IRAMFR/GILDAS
40 min per source and transition. HCNO (4-3) and (9-8) were observed in IRAS 16293-2422, but they were not detected after $1 \mathrm{~h}$ of on-source observing time.

\section{Results}

Cyanic acid ( $\mathrm{HOCN})$ has been detected towards three cold dense cores and the center of the low-mass "lukewarm" corino L1527 (see Fig. 1). Three rotational transitions were detected toward B1-b, which shows the strongest intensities, while in the cold cores L183 and L1544 two and one transitions were detected, respectively. The HOCN $\left(4_{04}-3_{03}\right)$ transition is marginally detected in the central position of L1527, while no lines were detected toward L1527 (20", $\left.-20^{\prime \prime}\right)$ with an rms of $9 \mathrm{mK}$, where one transition of HCNO has been previously observed (Marcelino et al. 2009). Although in some cases we only have one line to support the identification of HOCN in dark clouds, we are fully confident of the assignment as the density of lines in these sources is very low $(\leq 10$ lines/GHz), and the agreement in frequency between laboratory and astronomical observations is better than $20 \mathrm{kHz}$; i.e., near laboratory accuracy. Despite the non-detection of HCNO towards the cyanopolyyne peak of TMC-1, we have searched for HOCN to check the behavior of both isomers, but the $4_{04}-3_{03}$ transition was not detected with an rms of $6 \mathrm{mK}$. The observed line intensities are similar for HOCN and HCNO towards the dense cores observed in Marcelino et al. (2009), except for L1527, where the intensity of the $4_{04}-3_{03}$ transition of HOCN is half that of the HCNO $J=4-3$ one (see Fig. 1 in Marcelino et al. 2009). Since the dipole moments of cyanic and fulminic acid are similar (HOCN: $\mu_{a}=3.7 \mathrm{D}$, $\mu_{b}=1.6 \mathrm{D}$; HCNO: $\left.\mu=3.1 \mathrm{D}\right)$, i.e., the $a$-type line strengths of the observed transitions in the bent cyanic acid are very close to the observed line strengths of the linear fulminic acid, similar line intensities should reflect similar abundances (see below).

Three rotational transitions of cyanic acid (HOCN) were detected toward the low-mass, star-forming region IRAS 162932422. On the other hand, HCNO was not detected, with an observed rms of $10 \mathrm{mK}$ for the 4-3 line. Although each HOCN individual spectrum has a lower $\mathrm{S} / \mathrm{N}$ ratio than in the other sources, the three observed transitions show the same double-peaked line profile and consistent $V_{\text {LSR }}$ (lowest panels in Fig. 1), supporting a positive detection. IRAS $16293-2422$ is known to be a proto-binary system whose components, $\mathrm{A}$ and $\mathrm{B}$, separated by $5^{\prime \prime}$ (Wootten 1989), show a complex velocity pattern (see, e.g., Kuan et al. 2004). HNCO has been observed in IRAS 162932422 with emission at a systemic velocity of $2.5 \mathrm{~km} \mathrm{~s}^{-1}$ arising from both components but being more intense in component $\mathrm{A}$, which also shows a strong velocity feature at $V_{\mathrm{LSR}}=4.6 \mathrm{~km} \mathrm{~s}^{-1}$ (see Bisschop et al. 2008). It is therefore likely that most of the HOCN emission also comes from the A component. The doublepeaked profile has also been interpreted as arising from a disklike structure (see Huang et al. 2005; Stark et al. 2004, and references therein).

Four different transitions arising from fulminic acid (HCNO) were searched for in the SgrB2 molecular complex. The observed positions - SgrB2M, SgrB2M (20", 100"'), SgrB2N, and SgrB2S - were selected on the basis of previous detections of HOCN (see Brünken et al. 2010). However, no HCNO emission was found at any position, with rms values between $5-14 \mathrm{mK}$. Although a line was observed near $91.751 \mathrm{GHz}$, it has been recently identified as a transition arising from HSCN (see Halfen et al. 2009; Brünken et al. 2009b).

The three detected transitions towards B1-b and IRAS 16293-2422 arising from HOCN allow us to derive rotational 
Table 3. Derived column densities for HOCN and HCNO, and abundance ratios.

\begin{tabular}{|c|c|c|c|c|c|c|c|c|}
\hline Source & $\begin{array}{c}N(\mathrm{HOCN}) \\
10^{10} \mathrm{~cm}^{-2} \\
\end{array}$ & $\begin{array}{c}\mathrm{HOCN} / \mathrm{H}_{2}{ }^{a} \\
\times 10^{13} \\
\end{array}$ & $\begin{array}{c}N(\mathrm{HCNO}) \\
10^{10} \mathrm{~cm}^{-2} \\
\end{array}$ & $\begin{array}{c}\mathrm{HCNO} / \mathrm{H}_{2}{ }^{a} \\
\times 10^{13} \\
\end{array}$ & $\begin{array}{c}n\left(\mathrm{H}_{2}\right) \\
10^{5} \mathrm{~cm}^{-3} \\
\end{array}$ & $\mathrm{HNCO} / \mathrm{HOCN}$ & $\mathrm{HNCO} / \mathrm{HCNO}$ & $\mathrm{HCNO} / \mathrm{HOCN}$ \\
\hline B1-b & $14.3(6) / 10(1)$ & $11 / 7.7$ & $21(1) / 17(4)^{b}$ & $16 / 13$ & $20(5)$ & $62 / 80$ & $42 / 47$ & $1.5 / 1.7$ \\
\hline L1527 & $3.8(5) / 2.7(2)$ & $13 / 9.0$ & $6(1) / 4(1)^{b}$ & $21 / 15$ & $5(1)^{b}$ & $58 / 67$ & $34 / 40$ & $1.7 / 1.7$ \\
\hline L1527-B & $\leq 1 / 1.5$ & $\leq 3.3 / 5.0$ & $5(1) / 4(1)^{b}$ & $17 / 13$ & $5(1)^{b}$ & $\geq 90 / 53$ & $18 / 20$ & $\geq 5 / 2.7$ \\
\hline L1544 & $9(1) / 8.6(2)$ & $6.9 / 6.6$ & $6(3) / 6(2)^{b}$ & $4.6 / 4.8$ & $0.7(2)^{b}$ & $59 / 48$ & $88 / 65$ & $0.7 / 0.7$ \\
\hline L183 & $7(3) / 4.0(2)$ & $88 / 53$ & $6(2) / 5(1)^{b}$ & $75 / 66$ & $5(1)$ & $51 / 63$ & $60 / 50$ & $0.8 / 1.2$ \\
\hline TMC-1 & $\leq 0.9 / 1.9$ & $\leq 9 / 19$ & $\leq 1.3 / 1.4^{b}$ & $\leq 13 / 14$ & $0.3(1)^{b}$ & & & \\
\hline IRAS 16293-2422 & $125(4) / 160(1)$ & $3.6 / 4.6$ & $\leq 115 / 60$ & $\leq 3.3 / 1.7$ & $2.5(5)$ & $27 / 21$ & $\geq 30 / 57$ & $\leq 0.9 / 0.4$ \\
\hline SgrB2M-B & $2600^{c}$ & $\approx 260$ & $\leq 17$ & $\leq 1.7$ & $\approx 1.4^{a}$ & 181 & $\geq 30000$ & $\leq 0.006$ \\
\hline SgrB2M h.c. & $70000^{c}$ & $\approx 260$ & $\leq 5818$ & $\leq 22$ & $\approx 750^{a}$ & 90 & $\geq 1031$ & $\leq 0.083$ \\
\hline SgrB2M ext. & $750^{c}$ & $\approx 75$ & $\leq 14$ & $\leq 1.4$ & $\approx 1.4^{a}$ & 230 & $\geq 12143$ & $\leq 0.019$ \\
\hline SgrB2N h.c. & $670000^{c}$ & $\approx 5200$ & $\leq 79139$ & $\leq 609$ & $\approx 540^{a}$ & 313 & $\geq 2654$ & $\leq 0.118$ \\
\hline SgrB2N ext. & $1600^{c}$ & $\approx 160$ & $\leq 45$ & $\leq 4.5$ & $\approx 1.4^{a}$ & 181 & $\geq 6444$ & $\leq 0.028$ \\
\hline SgrB2S & $4200^{c}$ & $\approx 420$ & $\leq 40$ & $\leq 4.0$ & $\approx 1.4^{a}$ & 43 & $>4500$ & $<0.010$ \\
\hline
\end{tabular}

Notes. L1527-B corresponds to the position $\left(20^{\prime \prime},-20^{\prime \prime}\right)$ of this source, and SgrB2M-B to the offset $\left(20^{\prime \prime}, 100^{\prime \prime}\right)$ with respect to SgrB2M, h.c. refers to a hot core component, and ext. to the extended gas around this hot core. The first entry in the $N(\mathrm{HOCN}), N(\mathrm{HCNO})$ values, and in the abundances ratios corresponds to the column density obtained from the rotational diagrams, while the second one corresponds to the same parameter obtained from the $L V G$ calculations. Column densities in cold cores for HNCO are from Marcelino et al. (2009), and those for the GC clouds from Brünken et al. (2010). For IRAS 16293-2422 we have assumed $N(\mathrm{HNCO})=3.4 \times 10^{13}$ (van Dishoeck et al. 1995). ${ }^{a}$ Assumed $\mathrm{H}_{2}$ column densities are: B1-b: $1.3 \times 10^{23} \mathrm{~cm}^{-2}$ (Hirano et al. 1999); L1527: $3 \times 10^{22} \mathrm{~cm}^{-2}$ (Jørgensen et al. 2002); L1544: $1.3 \times 10^{23} \mathrm{~cm}^{-2}$ (Ward-Thompson et al. 1999); L183: 7.6 $\times 10^{21} \mathrm{~cm}^{-2}$ (Swade 1989); TMC-1(CP): $1 \times 10^{22} \mathrm{~cm}^{-2}$ (Cernicharo \& Guélin 1987); IRAS 16293-2422: $3.5 \times 10^{24} \mathrm{~cm}^{-2}$ (Bottinelli et al. 2004); SgrB2M-B, SgrB2S, SgrB2M ext., and SgrB2N ext.: $1 \times 10^{24} \mathrm{~cm}^{-2}$ (Lis \& Goldsmith 1990); SgrB2M h.c.: $2.7 \times 10^{25} \mathrm{~cm}^{-2}$ (Liu \& Snyder 1999); and SgrB2N h.c.: $1.3 \times 10^{25} \mathrm{~cm}^{-2}$ (Belloche et al. 2008). Source sizes for the GC clouds from Brünken et al. (2010), assumed distance to the GC clouds $8 \mathrm{kpc}$ (Reid 1993). ${ }^{b}$ From Marcelino et al. (2009). ${ }^{c}$ From Brünken et al. (2010).

temperatures of $T_{\text {rot }}=8.5 \pm 1.5 \mathrm{~K}$ and $11 \pm 1 \mathrm{~K}$ for B1-b and IRAS 16293-2422, respectively; in the latter, a source size of $10^{\prime \prime}$ has been assumed (van Dishoeck et al. 1995). With these temperatures, we obtained the column densities given in Table 3. For the other cold cores, those with HOCN detections and nondetections, we adopted the temperatures obtained for HNCO (see Marcelino et al. 2009) in order to compute column densities and upper limits to the column density (for the offset position of L1527 and TMC-1). The obtained upper limits to the column density and to the HNCO/HOCN abundance ratio in TMC- 1 are similar to those observed by Brünken et al. (2009a). For IRAS 16293-2422 and the GC molecular clouds we calculated upper limits to the column density of HCNO. In the former, we used the HOCN rotational temperature derived above, while for the latter we assumed the parameters (source sizes and temperatures) used by Brünken et al. (2010) for HOCN. Column densities, column density ratios, and fractional abundances for the assorted isomers are shown in Table 3 for cold cores and GC sources based on this work and prior studies. The fractional abundances for the GC sources are particularly uncertain mainly because of uncertain $\mathrm{H}_{2}$ column densities and densities. For Sgr B2N and Sgr $\mathrm{B} 2 \mathrm{M}$, we distinguished between the actual hot core and the extended gas surrounding the hot core. Our salient results are that the HOCN and HCNO column densities are similar in all the cold dense cores, while the warmer molecular clouds in SgrB2 show a very low abundance ratio for $\mathrm{HCNO} / \mathrm{HOCN}$.

To better constrain the observed column densities, we also performed LVG calculations. Since collisional rates for HOCN are not known, we adopted those of HNCO, as we did for HCNO (see Marcelino et al. 2009). We assumed a kinetic temperature of $10 \mathrm{~K}$ for all dark clouds. Although the derived rotational temperature for B1-b is lower, the observed intensities are better reproduced using $T_{\text {kin }}=10 \mathrm{~K}$. For both positions in L1527, we adopted a kinetic temperature of $15 \mathrm{~K}$ (see Marcelino et al. 2009). In the cores where only one transition was detected and also to obtain the upper limits for non detections, we used the volume density obtained in Marcelino et al. (2009). For IRAS 16293-2422, we used the temperature derived above from the rotational diagrams to calculate both the HOCN column density and the upper limit to that of HCNO. The derived column densities and ratios, together with the volume densities, are also shown in Table 3. The expected intensities obtained with the LVG aproximation for non-detected lines in L1544, L183, and L1527 $(0,0)$ are consistent with the observed rms noise in the spectra (see Fig. 1). The uncertainties in the LVG calculations are a factor 2 in density and $\pm 2 \mathrm{~K}$ in kinetic temperature. The corresponding errors in column density are dominated by the noise in the observations.

\section{Discussion}

The results shown in Table 3 indicate a change in the $\mathrm{HCNO} / \mathrm{HOCN}$ abundance ratio between cold clouds (quiescent or showing some indications of low-mass star formation activity) and sources in the GC, which are assumed to be warmer. In the latter, $\mathrm{HCNO}$ is not detected and the $\mathrm{HCNO} / \mathrm{HOCN}$ abundance ratio is $<10^{-1}-6 \times 10^{-3}$, while in cold clouds the ratio is close to unity (0.7-1.7). Nevertheless, the derived $\mathrm{HNCO} / \mathrm{HOCN}$ abundance ratio is between $20-320$ in all clouds. It therefore seems that the nature of the cloud does not influence the relative abundance of HOCN strongly with respect to the most abundant isomer HNCO. The situation is completely different for fulminic acid, HCNO. The abundance of HNCO with respect to fulminic acid was found by Marcelino et al. (2009) to range between 20 and 90 in cold clouds and to be much larger in warm clouds. This result is confirmed by the upper limits we obtain for this isomer towards the clouds in the GC.

We used two models to explain these results. The first is an extended pseudo-time-dependent approach based on the gas-phase network used in the steady-state results reported in 
Marcelino et al. (2009), while the second is a time-dependent, gas-grain approach (Hassel et al. 2008). In the pseudo-timedependent approach, the physical conditions are homogeneous and time-independent while the chemical process is occurring.

\subsection{Gas-phase model}

Little attention has been paid to gas-phase processes involving the elements $\mathrm{H}, \mathrm{N}, \mathrm{C}$, and $\mathrm{O}$ in the context of interstellar chemistry. Iglesias (1977) had a pioneer paper where chemical processes were proposed for NCO and HNCO. The major synthetic reaction for $\mathrm{NCO}$ is the reaction between $\mathrm{CN}$ and $\mathrm{O}_{2}$, which is sometimes referred to as a prototypical radical-radical reaction (Smith 1995) and has been studied from $13 \mathrm{~K}$ to $3720 \mathrm{~K}$. The NCO radical is a first step towards isocyanic acid and several of its isomers, which form by protonation, hydrogenation, and dissociative recombination. In our preceding paper (Marcelino et al. 2009), we considered exothermic neutral-neutral, ion-molecule, and dissociative recombination reactions to study the chemistry of $\mathrm{HNCO}, \mathrm{HOCN}$, and HCNO. Here, we reinvestigated the chemical processes and introduced the additional isomer isofulminic acid (HONC), which is the most energetic stable isomer of isocyanic acid, at an energy calculated to be $84.1 \mathrm{kcal} / \mathrm{mol}$ above that of HNCO (Schuurman et al. 2004), and whose rotational spectrum has recently been obtained in the laboratory (Mladenović et al. 2009). To preserve the consistency of the chemical network, we considered new ions arising from the protonation of $\mathrm{NCO}$ and $\mathrm{CNO}$, which may be progenitors of the four different CHNO isomers: i.e., $\mathrm{HNCO}^{+}, \mathrm{HOCN}^{+}, \mathrm{HCNO}^{+}$, and $\mathrm{HONC}^{+}$. The protonated $\mathrm{CHNO}$ ions have been theoretically studied by Ijjaali et al. (2001), and the present chemical network includes their 5 most stable structures $\mathrm{S} 1$ to $\mathrm{S} 5\left(\mathrm{H}_{2} \mathrm{NCO}^{+}\right.$, $\mathrm{HNCOH}^{+}, \mathrm{HCNOH}^{+}, \mathrm{H}_{2} \mathrm{OCN}^{+}$, and $\mathrm{H}_{2} \mathrm{CNO}^{+}$). These authors also determined transition states linking the stable protonated ions, among which two transition states, TS1-2 and TS3-5, connect stable isomers with transition energies of 95.2 and 172.5 $\mathrm{kcal} / \mathrm{mol}$, respectively. These values are obviously too high to play a role even in the warm environments of $\mathrm{Sg}$ B2.

Because little experimental information on the ion-molecule chemistry is available, we used the most conservative hypotheses by including only known exothermic channels and assuming that the structures of the reactants and products do not change dramatically in a chemical reaction. For example, in dissociative recombination reactions, we only allow those product channels in which the remaining heavy species has the same skeletal structure of the reactant ion upon separation of an $\mathrm{H}_{\text {or }} \mathrm{H}_{2}$; viz.,

$\mathrm{H}_{2} \mathrm{NCO}^{+}+\mathrm{e} \rightarrow \mathrm{HNCO}+\mathrm{H}$
$\mathrm{H}_{2} \mathrm{NCO}^{+}+\mathrm{e} \rightarrow \mathrm{NCO}+\mathrm{H}_{2}$
$\mathrm{HNCOH}^{+}+\mathrm{e} \rightarrow \mathrm{HNCO}+\mathrm{H}$
$\mathrm{HNCOH}^{+}+\mathrm{e} \rightarrow \mathrm{HOCN}+\mathrm{H}$
$\mathrm{HCNOH}^{+}+\mathrm{e} \rightarrow \mathrm{HCNO}+\mathrm{H}$
$\mathrm{HCNOH}^{+}+\mathrm{e} \rightarrow \mathrm{HONC}+\mathrm{H}$
$\mathrm{H}_{2} \mathrm{OCN}^{+}+\mathrm{e} \rightarrow \mathrm{HOCN}+\mathrm{H}$
$\mathrm{H}_{2} \mathrm{OCN}^{+}+\mathrm{e} \rightarrow \mathrm{NCO}+\mathrm{H}_{2}$
$\mathrm{H}_{2} \mathrm{CNO}^{+}+\mathrm{e} \rightarrow \mathrm{HCNO}+\mathrm{H}$.

When several product channels arise, we used the same value of the branching ratios in the absence of any theoretical or experimental argument.
Uniquely among the CHNO isomers, fulminic acid can possibly be formed by an efficient neutral-neutral reaction (Marcelino et al. 2009):

$$
\mathrm{CH}_{2}+\mathrm{NO} \rightarrow \mathrm{HCNO}+\mathrm{H}
$$

The reaction between $\mathrm{CH}_{2}$ and $\mathrm{NO}$ has been studied both theoretically and experimentally by a number of investigators, without a unique result concerning the product branching fractions. Some detailed studies show that HCNO is a dominant product (Roggenbuck \& Temps 1998; Fikri et al. 2001; Eschenko et al. 2002), while others do not (Zhang et al. 2004). In our standard model, based on the previous work of Marcelino et al. (2009), we consider HCNO to be a dominant product, while we do not in an alternative model. In general, the calculated abundances for $\mathrm{HCNO}$ in the alternative model are reduced by a wide range of factors from just a few to two orders of magnitude.

We display in Tables 4 (standard model) and 5 (alternative model) the fractional abundances relative to molecular hydrogen of the four CHNO isomers, as well as three isomeric ratios for a variety of different densities at two kinetic temperatures $(10 \mathrm{~K}$ and $50 \mathrm{~K}$ ). These temperatures represent, respectively, those of a cold dark cloud and the envelope of a hot core. Results are listed both for so-called early time, a time of $\leq 0.1 \mathrm{Myr}$, at which the HNCO abundance peaks, and at steady-state, which are reached around $10 \mathrm{Myr}$. We also ran the models at $200 \mathrm{~K}$ (hot core case), but the results do not differ much from those obtained at $50 \mathrm{~K}$ at the densities used, which do not range as high as our estimated central hot core densities in Table 3. We used the early-time results in comparison with observation, and the former are higher than the steady-state values by up to a factor of $\approx 5-10$.

In these models, the cosmic ionization rate is taken as $5 \times$ $10^{-17} \mathrm{~s}^{-1}$, and elemental abundances corresponding to the "low metal" case have been used. Some of the results for the lowest densities are unusual in that they are characteristic of the high ionization phase (HIP) (Le Bourlot et al. 1993), with vanishingly low values for saturated molecules, so the displayed ratios are not significant. The other results are characteristic of the better known, more standard low ionization phase.

The fractional abundances calculated for HNCO and HOCN for the cold sources tend to be too high by an order of magnitude or so, while for HCNO this is true only for the standard model. The results of the alternative model, on the other hand, underestimate the observed HCNO fractional abundances in the cold sources. In the GC sources, the $\mathrm{H}_{2}$ column densities and densities are more uncertain, but it appears that the $50 \mathrm{~K}$ theoretical results at a density of $1 \times 10^{5} \mathrm{~cm}^{-3}$ for $\mathrm{HNCO}$ and HOCN reproduce the hot core envelopes well, but the higher-density results are significantly lower than the observed values for the hot cores themselves.

If we focus on the abundance ratios among the HNCO isomers, we note that the observed $\mathrm{HNCO} / \mathrm{HOCN}$ ratio in the cold clouds $(\sim 50-100)$ is fit closely at the appropriate densities by the early-time theoretical results, whereas the $\mathrm{HCNO} / \mathrm{HOCN}$ ratio $(\sim 1)$ is fit in the standard case but is underproduced by the alternative case. We conclude that the standard model is needed and that HCNO must be produced in the gas-phase model by the reaction between $\mathrm{CH}_{2}$ and $\mathrm{NO}$.

In the GC clouds and IRAS 16293-2422, the HNCO/HOCN ratio is also reproduced reasonably well. On the other hand, the low $\mathrm{HCNO} / \mathrm{HOCN}$ ratios are not reproduced by the standard model, which tends to be 1-2 orders of magnitude higher. The alternative model, on the other hand, does somewhat better. This conflict between the standard and alternative models, depending 
Table 4. Fractional abundances and abundance ratios from the standard gas-phase model ${ }^{a}$.

\begin{tabular}{|c|c|c|c|c|c|c|c|}
\hline$T=10 \mathrm{~K}$ & $\begin{array}{l}\mathrm{HNCO} / \mathrm{H}_{2} \\
\times 10^{13} \\
\end{array}$ & $\begin{array}{l}\mathrm{HOCN} / \mathrm{H}_{2} \\
\times 10^{13} \\
\end{array}$ & $\begin{array}{l}\mathrm{HCNO} / \mathrm{H}_{2} \\
\times 10^{13} \\
\end{array}$ & $\begin{array}{l}\mathrm{HONC} / \mathrm{H}_{2} \\
\times 10^{13}\end{array}$ & $\mathrm{HNCO} / \mathrm{HOCN}$ & $\mathrm{HNCO} / \mathrm{HCNO}$ & $\mathrm{HCNO} / \mathrm{HOCN}$ \\
\hline \multicolumn{8}{|l|}{$n\left(\mathrm{H}_{2}\right) \mathrm{cm}^{-3}$} \\
\hline \multirow[t]{2}{*}{$1 \times 10^{3}$} & 3.3 & 0.67 & 5.1 & 1.5 & 5.0 & 0.6 & 7.7 \\
\hline & 4.7 & 1.0 & 7.1 & 2.0 & 4.7 & 0.7 & 7.1 \\
\hline \multirow[t]{2}{*}{$3 \times 10^{3}$} & 9000 & 720 & 2000 & 380 & 13 & 4.6 & 2.7 \\
\hline & 20000 & 1400 & 4900 & 1100 & 14 & 4.1 & 3.5 \\
\hline \multirow[t]{2}{*}{$1 \times 10^{4}$} & 19000 & 1300 & 2100 & 170 & 14 & 8.9 & 1.6 \\
\hline & 47000 & 2300 & 4500 & 400 & 20 & 11 & 1.9 \\
\hline \multirow[t]{2}{*}{$3 \times 10^{4}$} & 18000 & 1200 & 1300 & 41 & 15 & 13 & 1.1 \\
\hline & 55000 & 1900 & 2500 & 90 & 30 & 22 & 1.3 \\
\hline \multirow[t]{2}{*}{$1 \times 10^{5}$} & 9200 & 670 & 680 & 6.8 & 14 & 14 & 1.0 \\
\hline & 41000 & 980 & 1000 & 11 & 42 & 39 & 1.1 \\
\hline \multirow[t]{2}{*}{$3 \times 10^{5}$} & 3400 & 300 & 380 & 1.3 & 11 & 9.0 & 1.3 \\
\hline & 20000 & 420 & 440 & 1.5 & 47 & 45 & 1.1 \\
\hline \multirow[t]{2}{*}{$5 \times 10^{5}$} & 2000 & 190 & 290 & 0.6 & 10 & 6.7 & 1.5 \\
\hline & 13000 & 290 & 340 & 0.8 & 45 & 38 & 1.2 \\
\hline \multirow[t]{2}{*}{$10^{6}$} & 900 & 100 & 200 & 0.2 & 8.7 & 4.5 & 1.9 \\
\hline & 7100 & 160 & 180 & 0.2 & 46 & 39 & 1.2 \\
\hline$T=50 \mathrm{~K}$ & $\begin{array}{l}\mathrm{HNCO} / \mathrm{H}_{2} \\
\times 10^{13}\end{array}$ & $\begin{array}{l}\mathrm{HOCN} / \mathrm{H}_{2} \\
\times 10^{13}\end{array}$ & $\begin{array}{l}\mathrm{HCNO} / \mathrm{H}_{2} \\
\times 10^{13}\end{array}$ & $\begin{array}{l}\mathrm{HONC} / \mathrm{H}_{2} \\
\times 10^{13}\end{array}$ & $\mathrm{HNCO} / \mathrm{HOCN}$ & $\mathrm{HNCO} / \mathrm{HCNO}$ & $\mathrm{HCNO} / \mathrm{HOCN}$ \\
\hline \multicolumn{8}{|l|}{$n\left(\mathrm{H}_{2}\right) \mathrm{cm}^{-3}$} \\
\hline \multirow[t]{2}{*}{$1 \times 10^{3}$} & 16000 & 240 & 5200 & 2100 & 66 & 3.1 & 22 \\
\hline & 24000 & 330 & 8500 & 4000 & 74 & 2.8 & 26 \\
\hline \multirow[t]{2}{*}{$1 \times 10^{4}$} & 32000 & 650 & 2400 & 220 & 49 & 13 & 3.8 \\
\hline & 110000 & 1700 & 3300 & 240 & 63 & 33 & 1.9 \\
\hline \multirow[t]{2}{*}{$1 \times 10^{5}$} & 9600 & 240 & 380 & 4.1 & 40 & 25 & 1.6 \\
\hline & 49000 & 670 & 180 & 1.4 & 73 & 280 & 0.3 \\
\hline \multirow[t]{2}{*}{$1 \times 10^{6}$} & 1000 & 17 & 36 & 0.04 & 59 & 28 & 2.1 \\
\hline & 6700 & 22 & 7.0 & 0.005 & 300 & 960 & 0.3 \\
\hline
\end{tabular}

Notes. For each density and temperature, the first line refers to steady state whereas the second line refers to early time. Early-time results computed at maximum of $\mathrm{HNCO}$ abundance. ${ }^{(a)}$ With the rate coefficients for $\mathrm{CH}_{2}+\mathrm{NO}$ measured by Fikri et al. (2001), in which $\mathrm{HCNO}$ is the major product.

upon whether the clouds are cold or warm, suggests that more detailed gas-grain models should be considered, which for warm sources include the actual warm-up stage.

\subsection{Gas-grain model}

To simulate the different regions where the CHNO isomers are detected, we utilized the Ohio State gas-grain network (Garrod \& Herbst 2006; Garrod et al. 2008) with a variety of physical conditions. In this code, both gas-phase and grain-surface chemistry are included, and solutions are found as functions of time. Even more so than in the pure gas-phase modeling discussed above, many of the rate coefficients involving these isomers are estimated. In addition to a model for a cold core at $10 \mathrm{~K}$ and an $\mathrm{H}_{2}$ density of $10^{4} \mathrm{~cm}^{-3}$, we computed three different warm-up models, which undergo a cold phase $(10 \mathrm{~K})$ that lasts for $10^{5} \mathrm{yr}$ followed by a warm-up that lasts $2 \times 10^{5} \mathrm{yr}$ where the temperature increases quadratically with time (Viti et al. 2004) to an asymptotic value depending on the physical object being simulated. The asymptotic temperatures for a lukewarm corino (e.g. L1527), a warm envelope surrounding a hot core, and the hot core itself are $30 \mathrm{~K}, 50 \mathrm{~K}$, and $200 \mathrm{~K}$, respectively (Garrod \& Herbst 2006; Hassel et al. 2008). The $\mathrm{H}_{2}$ densities in the three models are $10^{6}, 10^{5}$, and $10^{6} \mathrm{~cm}^{-3}$, respectively. As in the gasphase calculations, two models have been run, depending upon the products of the $\mathrm{CH}_{2}+\mathrm{NO}$ reaction. We once again use the terms "standard" and "alternative" for the two models.
In general, the results of the gas-grain model calculations do not totally solve the theoretical problem of accounting for the comparable abundances of HCNO and HOCN in cold regions and the much lower abundance of $\mathrm{HCNO}$ with respect to HOCN in warmer ones. Since the gas-grain results will be the subject of a future paper (Quan, Osamura, \& Herbst, in preparation), we concentrate here on one type of warm-up model, the warm envelope surrounding a hot core.

Figure 2 shows calculated gas-phase fractional abundances for the four CHNO isomers in the gas phase as functions of time for the standard and alternative warm-up envelope models. At times up to $10^{5} \mathrm{yr}$, the calculated abundances are similar to those of the cold core model. Here, the HCNO isomer is predicted to be significantly more abundant than $\mathrm{HOCN}$, in disagreement with observations. During the subsequent warm-up period, the abundances of $\mathrm{HNCO}$ and HOCN increase markedly, whereas those of HCNO and HONC do not. Still, at the start of the warm-up era, there is little HOCN, so that despite its subsequent increase, it achieves a higher abundance than HCNO only for a brief period. After the asymptotic temperature of $50 \mathrm{~K}$ is reached, all abundances decrease sharply or gradually, and the abundance of HCNO remains higher than that of HOCN, even with the alternative model. During the brief period near the time of peak abundances, which occurs near the end of the warmup period, the fractional abundance of HNCO equals (alternative model) or exceeds (standard model) $10^{-8}$, while those of HOCN and HCNO lie in the vicinity of $10^{-11}$ and $10^{-11}-10^{-12}$, respectively. The peak calculated abundance of $\mathrm{HNCO}$ exceeds 
Table 5. Fractional abundances and abundance ratios from the alternative gas-phase model $^{a}$.

\begin{tabular}{|c|c|c|c|c|c|c|c|}
\hline$T=10 \mathrm{~K}$ & $\begin{array}{l}\mathrm{HNCO} / \mathrm{H}_{2} \\
\times 10^{13}\end{array}$ & $\begin{array}{l}\mathrm{HOCN} / \mathrm{H}_{2} \\
\times 10^{13}\end{array}$ & $\begin{array}{l}\mathrm{HCNO} / \mathrm{H}_{2} \\
\times 10^{13}\end{array}$ & $\begin{array}{l}\mathrm{HONC} / \mathrm{H}_{2} \\
\times 10^{13}\end{array}$ & $\mathrm{HNCO} / \mathrm{HOCN}$ & $\mathrm{HNCO} / \mathrm{HCNO}$ & $\mathrm{HCNO} / \mathrm{HOCN}$ \\
\hline \multicolumn{8}{|l|}{$n\left(\mathrm{H}_{2}\right) \mathrm{cm}^{-3}$} \\
\hline \multirow[t]{2}{*}{$1 \times 10^{3}$} & 3.5 & 0.72 & 4.6 & 1.0 & 4.9 & 0.80 & 6.4 \\
\hline & 1000 & 91 & 280 & 170 & 11 & 3.6 & 3.1 \\
\hline \multirow[t]{2}{*}{$3 \times 10^{3}$} & 9500 & 850 & 630 & 180 & 11 & 15 & 0.7 \\
\hline & 21000 & 1700 & 1200 & 550 & 12 & 17 & 0.7 \\
\hline \multirow[t]{2}{*}{$1 \times 10^{4}$} & 19000 & 1500 & 330 & 45 & 13 & 58 & 0.2 \\
\hline & 48000 & 2700 & 630 & 120 & 18 & 76 & 0.2 \\
\hline \multirow[t]{2}{*}{$3 \times 10^{4}$} & 18000 & 1300 & 96 & 4.6 & 14 & 190 & 0.07 \\
\hline & 55000 & 2100 & 190 & 12 & 27 & 300 & 0.09 \\
\hline \multirow[t]{2}{*}{$1 \times 10^{5}$} & 9400 & 730 & 19 & 0.2 & 13 & 490 & 0.03 \\
\hline & 41000 & 1100 & 40 & 0.6 & 37 & 1000 & 0.04 \\
\hline \multirow[t]{2}{*}{$3 \times 10^{5}$} & 3400 & 330 & 4.4 & 0.02 & 10 & 770 & 0.01 \\
\hline & 20000 & 490 & 11 & 0.05 & 40 & 1800 & 0.02 \\
\hline \multirow[t]{2}{*}{$5 \times 10^{5}$} & 2000 & 220 & 2.3 & 0.005 & 8.9 & 850 & 0.01 \\
\hline & 13000 & 320 & 6.4 & 0.02 & 40 & 2000 & 0.02 \\
\hline \multirow[t]{2}{*}{$10^{6}$} & 900 & 120 & 1.0 & 0.001 & 7.3 & 910 & 0.01 \\
\hline & 7100 & 170 & 2.9 & 0.003 & 41 & 2400 & 0.02 \\
\hline$T=50 \mathrm{~K}$ & $\begin{array}{l}\mathrm{HNCO} / \mathrm{H}_{2} \\
\times 10^{13}\end{array}$ & $\begin{array}{l}\mathrm{HOCN} / \mathrm{H}_{2} \\
\times 10^{13}\end{array}$ & $\begin{array}{l}\mathrm{HCNO} / \mathrm{H}_{2} \\
\times 10^{13} \\
\end{array}$ & $\begin{array}{l}\mathrm{HONC} / \mathrm{H}_{2} \\
\times 10^{13}\end{array}$ & $\mathrm{HNCO} / \mathrm{HOCN}$ & $\mathrm{HNCO} / \mathrm{HCNO}$ & $\mathrm{HCNO} / \mathrm{HOCN}$ \\
\hline \multicolumn{8}{|l|}{$n\left(\mathrm{H}_{2}\right) \mathrm{cm}^{-3}$} \\
\hline \multirow[t]{2}{*}{$1 \times 10^{3}$} & 17000 & 590 & 2000 & 1100 & 30 & 8.8 & 3.4 \\
\hline & 26000 & 930 & 3100 & 2300 & 28 & 8.4 & 3.3 \\
\hline \multirow[t]{2}{*}{$1 \times 10^{4}$} & 32000 & 840 & 470 & 66 & 38 & 68 & 0.6 \\
\hline & 110000 & 1900 & 970 & 110 & 56 & 110 & 0.5 \\
\hline \multirow[t]{2}{*}{$1 \times 10^{5}$} & 9600 & 260 & 26 & 0.4 & 37 & 370 & 0.1 \\
\hline & 49000 & 720 & 56 & 0.6 & 68 & 880 & 0.08 \\
\hline \multirow[t]{2}{*}{$1 \times 10^{6}$} & 1000 & 18 & 1.0 & 0.001 & 57 & 990 & 0.06 \\
\hline & 6700 & 25 & 2.3 & 0.002 & 270 & 2900 & 0.09 \\
\hline
\end{tabular}

Notes. For each density and temperature, the first line refers to steady state whereas the second line refers to early time. Early-time results computed at maximum of HNCO abundance. ${ }^{(a)}$ With the rate coefficients for $\mathrm{CH}_{2}+\mathrm{NO}$ calculated by Zhang et al. (2004), in which $\mathrm{HCNO}$ is not a major product. See text.

its observed value, while that of $\mathrm{HOCN}$ reasonably agrees with observation (see Fig. 2). The predicted minimum HCNO/HOCN ratio is $\approx 0.5$ for the standard case and $\approx 0.1$ for the alternative one; the latter is in closer agreement with observations.

The observed higher upper limit for the HCNO/HOCN ratio in IRAS 16293-2422, which is similar in temperature to the environs of a hot core, is reproduced slightly before or slightly after the peak abundances in our envelope model, after which the HCNO abundance becomes larger than for HOCN. In the standard model, an abundance ratio of unity occurs at 2 and $4 \times 10^{5} \mathrm{yr}$, while in the alternative model, such an abundance ratio occurs at slightly later times.

\section{Conclusions}

In this paper we have presented observations of the HNCO metastable isomers, HOCN and HCNO, in several sources including quiescent prestellar cores, low-mass protostellar objects, and hot cores and their environs, in particular the GC sources. While HOCN was observed in all sources (except TMC-1) with a similar $\mathrm{HNCO} / \mathrm{HOCN}$ abundance ratio, $\mathrm{HCNO}$ was not detected towards the low-mass protostar IRAS 16293-2422 or the warm GC clouds. Because HCNO is higher in energy than HOCN, this behavior of the CHNO isomers is similar to that of $\mathrm{HCN}$ and $\mathrm{HNC}$, where the latter seems to be depleted when the kinetic temperature of the cloud increases.

In an attempt to explain the fractional and relative abundances of the CHNO isomers, we used both a gas-phase,

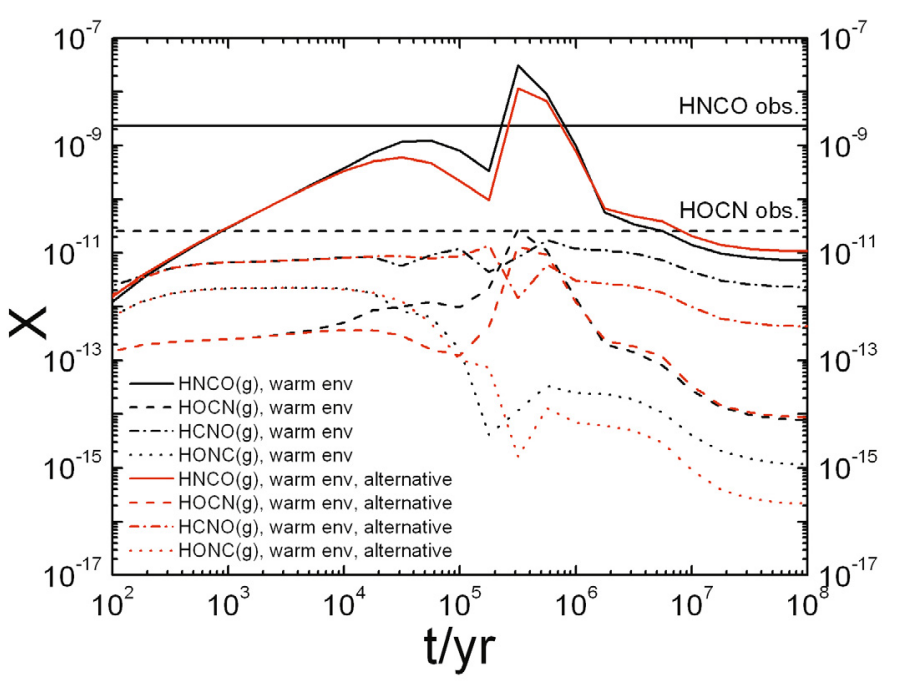

Fig. 2. Fractional abundances $(X)$ for the gaseous $\mathrm{CHNO}$ isomers with respect to molecular hydrogen computed with the gas-grain model for the case of a warm envelope surrounding a hot core. The observed values are in the vicinity of those observed for the extended environments considered here.

pseudo-time-dependent model and a gas-grain model that specifically considers a warm-up phase in between cold and warm eras. The results of both models are mixed and, for HCNO, 
depend to some extent on whether or not the gas-phase reaction between $\mathrm{CH}_{2}$ and $\mathrm{NO}$ produces $\mathrm{HCNO}$.

In summary, much of the chemistry is still poorly understood, and it is not clear in particular why HCNO is so underabundant with respect to HOCN in the warm sources but not in the cold ones. It is probable that we are missing an important destruction mechanism for HCNO. Although we considered several destruction channels (in particular reactions with atomic $\mathrm{C}$, $\mathrm{O}$, and $\mathrm{H}$ ), we are not able to reproduce the low observed abundances at high temperatures. One possibility would be a mechanism that converts $\mathrm{HCNO}$ into $\mathrm{HOCN}$, in analogy to the conversion of $\mathrm{HNC}$ into $\mathrm{HCN}$ in warm sources.

Even so, the chemistry of the CHNO isomers that occurs on the dust grains may play a key role in the relative abundances of $\mathrm{HNCO} / \mathrm{HCNO} / \mathrm{HOCN}$. Laboratory experiments on the surface formation of these isomers via hydrogenation of $\mathrm{NCO}$ and $\mathrm{CNO}$ could add quantitative and qualitative information to our models. One basis of both our gas-phase and gas-grain chemistry is the radical NCO. Detection or non-detection of this species in space would also help to constrain our chemical models.

Acknowledgements. We thank the referee for pointing out additional information on the reaction between $\mathrm{CH}_{2}$ and $\mathrm{NO}$ and for other useful comments. We thank M. Gerin for useful discussions concerning the chemistry. This work has been supported by Spanish Ministerio de Ciencia e Innovación through grants AYA2006-14876 and ESP2007-65812-CO2-01, and by DGU of the Madrid community government under IV-PRICIT project S-0505/ESP-0237 (ASTROCAM). E.H. acknowledges the support of the National Science Foundation for his astrochemistry program through grant AST-0702876 and for his program on chemical kinetics through the Center for the Chemistry of the Universe. E.H. also acknowledges the support of NASA for studies in the evolution of pre-planetary matter.

\section{References}

Bisschop, S. E., Jørgensen, J. K., Bourke, T. L., Bottinelli, S., \& van Dishoeck, E. F. 2008 , A\&A, 488, 959

Belloche, A., Menten, K. M., Comito, C., et al. 1990, A\&A, 482, 179

Bottinelli, S., Ceccarelli, C., Neri, R., et al. 2004, ApJ, 617, 69

Brünken, S., Gottlieb, C. A., McCarthy, M. C., \& Thaddeus, P. 2009a, ApJ, 697, 880

Brünken, S., Yu, Z., Gottlieb, C. A., McCarthy, M. C., \& Thaddeus, P. 2009b, ApJ, 706, 1588
Brünken, S., Belloche, A., Martín, S., Verheyen, L., \& Menten, K. M. 2010, A\&A, 516, A109

Cazaux, S., Tielens, A. G. G. M., Ceccarelli, C., et al. 2003, ApJ, 593, 51

Cernicharo, J. 1985, IRAM report No. 52 (Granada: IRAM)

Cernicharo J., \& Guélin M. 1987, A\&A, 176, 299

Churchwell, E., Nash, A. G., \& Walmsley, C. M. 1984, ApJ, 287, 681

Eschenko, G., Koecher, T., Kerst, C., \& Temps, F. 2002, Chem. Phys. Lett., 356, 181

Fikri, M., Meyer, S., Roggenbuck, J., \& Temps, F. 2001, Faraday Disc., 119, 223 Garrod, R. T., \& Herbst, E. 2006, A\&A, 457, 927

Garrod, R. T., Widicus Weaver, S. L, \& Herbst, E. 2008, ApJ, 682, 283

Halfen, D. T., Ziurys, L. M., Brünken, S., et al. 2009, ApJ, 702, L124

Hassel, G. E., Herbst, E., \& Garrod, R. T. 2008, ApJ, 681, 1385

Herbst, E., Terzieva, R., \& Talbi, D. 2000, MNRAS, 311, 869

Hirano, N., Kamazaki, T., Mikami, H., Ohashi, N., \& Umemoto, T. 1999, in Star Formation 1999, ed. T. Nakamoto, NRO, 181

Hirota, T., Yamamoto, S., Mikami, H., \& Ohishi, M. 1998, ApJ, 503, 717

Huang, H.-C., Kuan, Y.-J., Charnley, S. B., et al. 2005, Adv. Space Res., 36, 146 Iglesias E. 1977, ApJ, 218, 697

Ijjaali, F., Alcami, M., Mó, O., \& Yáñez, M. 2001, Mol. Phys., 99, 1129

Irvine, W. M., \& Schloerb, F. P. 1984, ApJ, 282, 516

Jørgensen, J. K., Schöier, F. L., \& van Dishoeck, E. F. 2002, A\&A, 389, 908

Kuan, Y.-J., Huang, H.-C., Charnley, S. B., et al. 2004, ApJ, 616, 27

Le Bourlot, J., Pineau des Forets, G., Roueff, E., \& Schilke, P. 1993, ApJ, 416, L87

Lis, D. C., \& Goldsmith, P. F. 1990, ApJ, 356, 195

Liu, S.-Y., \& Snyder, L. E. 1999, ApJ, 523, 683

Marcelino, N., Cernicharo, J., Tercero, B., \& Roueff, E. 2009, ApJ, 690, 27

Mladenović M., Lewerenz M., McCarthy M. C., \& Thaddeus P. 2009, J. Chem. Phys., 131, 174308

Reid, M. J. 1993, ARA\&A, 31, 345

Roggenbuck, J., \& Temps, F. 1998, Chem. Phys. Lett., 285, 422

Schilke, P., Walmsley, C. M., Pineau des Forêts, G., et al. 1992, A\&A, 256, 595

Schuurman M. S., Muir, S. R., Allen W. D., \& Shaefer III H. F. 2004, J. Chem. Phys., 120, 11586

Smith I. W. M. 1995, Int. J. Mass. Spectr. Ion Proc., 149, 231

Stark, R., Sandell, G., Beck, S. C., et al. 2004, ApJ, 608, 341

Swade, D. A. 1989, ApJ, 345, 828

Talbi, D., Ellinger, Y., \& Herbst, E. 1996, A\&A, 314, 688

van Dishoeck, E. F., Blake, G. A., Jansen, D. J., \& Groesbeck, T. D. 1995, ApJ, 447,760

Viti, S., Collings, M. P., Dever, J. W., McCoustra, M. R. S., \& Williams, D. A. 2004, MNRAS, 354, 1141

Ward-Thompson, D., Motte, F., \& André, P. 1999, MNRAS, 305, 143

Watson, W. D., \& Walmsley, C. M. 1982, in Regions of Recent Star Formation, ed. R. S. Roger, \& E. Dewdney (Dordrecht: Reidel), 357

Wootten, A. 1989, ApJ, 337, 858

Zhang, W., Du, B., \& Feng, C. 2004, J. Mol. Struc. (Theochem), 679, 121 